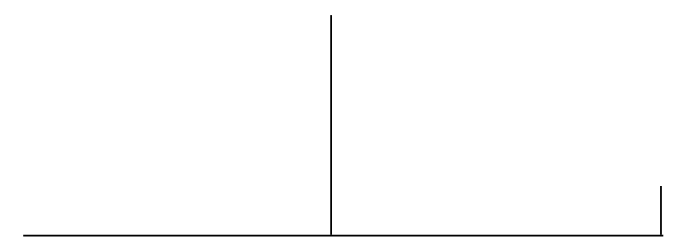

Rev. Latinoam. Psicopat. Fund., São Paulo, v. 13, n. 2, p. 265-282, junho 2010

Ajai Singh

\title{
Medicina moderna: rumo à prevenção, à cura, ao bem-estar e à longevidade*
}

\begin{abstract}
Apresentação
O artigo que "Observando a Medicina" traduz nesta edição traz um ponto de vista surpreendente: o de que a "medicina moderna" - como chamada pelo autor, o psiquiatra Ajai Singh, de Mumbai, India - deve corrigir seu rumo, de uma medicina que busca o alívio da doença, para uma medicina que busque a cura e a prevenção. $\mathrm{O}$ artigo chama a atenção para uma insatisfação com a medicina medicamentosa voltada para a estabilização, gerando uma certa preguiça de fazer pesquisa visando a prevenção e a cura. Para Singh, a ênfase em tratamentos paliativos identificados com controle - é uma manifestação de Tanatos, enquanto a medicina deveria buscar "tornar-se a mais gloriosa manifestação de um Eros que previne/cura doenças". O que importa, diz ele, é o intervalo anterior ao final - quando, afinal, Tanatos prevalecerá.
\end{abstract}

* Disponível em <http://www.msmonographs.org>.

Tradução de Luana Villac. 


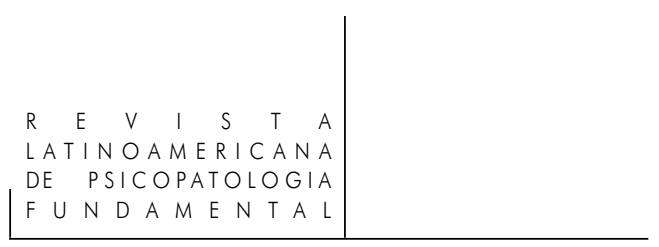

Para que o intervalo seja erótico, argumenta, a prioridade da medicina deveria ser evitar que se chegue aos hospitais e clínicas, o que não ocorre porque as ações importantes para a prevenção - entre outras, "água limpa, alimentação nutritiva, moradias limpas e em locais seguros, saneamento apropriado, (...) maior poder aos desprovidos de recursos" - estão fora do "controle da medicina; e cortariam pela "raiz a justificativa para o establishment médico e sua proliferação". Entre as medidas de prevenção, Singh inclui a pesquisa de vacinas, não apenas para doenças infecciosas, mas também para doenças desde o diabetes até a esquizofrenia. Segundo menciona no artigo, já há sugestões dessas vacinas, também para outras doenças mentais. Igualmente o papel da medicina comportamental, diz ele, "requer apoio entusiasta como um meio de promover comportamentos salutares". Ainda no tópico da prevenção, o autor preconiza que a medicina complementar e alternativa seja submetida a "rigoroso exame experimental e científico". Segue-se a esta observação uma lista de referências que o leitor poderá pesquisar.

A segunda prioridade da medicina erótica do doutor Singh é a busca da cura. Se alguém chega ao hospital, diz ele, é tarefa dessa medicina fazer com que a pessoa não tenha a mesma doença novamente nem "outra relacionada com ela'. O psiquiatra observa que, embora ele classifique como "distante" a possibilidade da cura, "distante não significa inalcançável". Como terceira prioridade, ele apela para que a medicina estude "de perto" a longevidade e o bem-estar; nestes tópicos, apresenta uma lista de referências às quais dirige seu entusiasmo, em especial à pesquisa com grupos de pessoas com cem anos ou mais.

Em tempos de elogio frequentemente superficial às consequências, para a medicina, das informações obtidas sobre o genoma dos organismos, é um ponto de vista que pode ensejar o contraditório. Mr. Singh é editor do site Mens Sana Monographs, onde foi encontrado o artigo traduzido. No mesmo site, há uma biografia em que está registrado ter sido ele vice-presidente e presidente da Associação Indiana de Psiquiatria Privada, para o estado de Maharashtra; e ser o presidente fundador de "uma organização dedicada a converter cantores de banheiro em cantores de palco". Mr. Singh é bem-humorado.

Mônica Teixeira 
A medicina moderna tem realizado muitas coisas nos campos de doenças infecciosas e de emergências para assistir a cura. Na maior parte dos outros campos, ela visa, sobretudo, ao controle, que é outro nome para o tratamento paliativo. A farmacologia, na qual se inclui a psicofarmacologia, é igualmente dirigida ao controle e ao alívio. Está na hora de profissionais da área e pesquisadores voltarem-se decisivamente para a prevenção e para a cura. Ademais, o outro grande desafio da medicina moderna é a longevidade aliada ao bem-estar. Avanços em vacinas contra hipertensão, diabetes, câncer etc. merecem atenção, bem como o papel da meditação, yoga, espiritualidade etc. na prevenção de doenças em vários níveis. Pesquisas sobre longevidade, mudanças no estilo de vida e centenários saudáveis merecem um exame especialmente minucioso na busca dos fatores que contribuem para a longevidade com bem-estar. Um olhar de perto para a medicina complementar e alternativa é necessário para encontrar modelos apropriados que esta possa vir a ter, à parte seus grandes discursos ou sua hostilidade para com o cuidado médico tradicional. A medicina é uma manifestação do Eros humano, e não deve tornar-se instrumento de seu Tanatos. Ela deve realizar seu verdadeiro potencial, de forma que Eros prevaleça, e Tanatos prevaleça apenas finalmente, não prematuramente.

Palavras-chave: Medicina, Eros, Tanatos, doença, prevenção, cura, paliação, longevidade, bem-estar, centenários, meditação, yoga, medicina complementar e alternativa, farmacologia, psicofarmacologia

Introdução

Eu acordei com um sonho sobre a medicina e fiquei pensando vagamente em analisá-lo. Então pensei, puxa, para quê? Freud já não está entre nós e a psicanálise não é moeda corrente. De qualquer forma, a vida está caminhando descansadamente em seu passo agradável. Sem maiores problemas. Então para que ficar escavando?

Mas a compreensão de um sonho da manhã precedente permanece em minha mente. Deixe-me contar-lhes um pouco sobre isso. A medicina moderna progrediu, e nós devemos agradecer toda a comunidade de pesquisadores e clínicos por isso. Mas também progrediu a patologia. O número de pessoas doentes não diminuiu, assim como seu sofrimento. A quantidade de doenças e de pacientes não está se reduzindo, está somente mudando de tipo (Singh e Singh, 2005). Não há dúvidas de que estendemos a longevidade, tornamos a terceira idade mais vivível, reduzimos a mortalidade infantil, tornamos o cotidiano mais livre de dor, graças às numerosas medicinas e procedimentos de nosso arsenal. Entretanto, nós não reduzimos o número de doentes e nem encontramos a cura para nenhuma doen- 
ça, a não ser as infecciosas, além de certamente termos feito progresso notáveis no atendimento de emergência. (Ambos, grandes mobilizações de recursos e de energia no sentido de reduzir o sofrimento social. Assim como os animais, o humano incluído, vão lutar e esforçar-se para enfrentar as emergências - especialmente em casos de risco de vida ou danos a membros do corpo - o animal da sociedade desenvolveu métodos eficazes de fazer face às suas emergências. Com isso, métodos para atacar infecções e problemas médicos/cirúrgicos agudos são, de fato, eficientes).

No caso da maior parte de outros tipos de cuidados médicos, o foco é principalmente o controle - desacelerar o avanço, reduzir a dor, minimizar as deficiências, adiar a morte.

Está fora de moda pensar em termos de cura, como se essa fosse uma palavra odiosa.

\section{A medicina moderna é, sobretudo, paliativa}

A medicina moderna é, sobretudo, paliativa e bastante orgulhosa de sê-la. Não estou me referindo ao câncer. Por tratamento paliativo eu me refiro àquele que reduz ou ameniza a severidade da dor ou da doença sem remover sua causa e nem efetuar uma cura (do latim palliare, encobrir).

Trata-se, em outras palavras, de controle.

Mais precisamente, é como transformar uma necessidade em virtude. E também, falsa bravata.

O que a medicina precisa fazer é impelir-se em direção à cura, por um lado, e à prevenção, por outro. A cura, todavia, está fora de questão, já que parece inalcançável, e a prevenção não está em lugar nenhum da consciência coletiva de pesquisadores biomédicos e de formadores de opinião. Eu suspeito que provavelmente isso se dê porque elas findariam por torná-los redundantes. E o lobby da prevenção e da cura faz apenas barulhos fracos, se é que faz algum, e não possui o poder de mudar a opinião dominante.

O que isso realmente significa é que enquanto a verdade está lá para ser vista por todos e agir sobre seu trabalho, nada é feito. Por que deve ser assim? Será que é porque a humanidade é guiada por um Tanatos arrojadamente dirigido, conforme afirmou Freud? De modo que ela nega e impede realizações importantes, pois deve impelir-se rumo ao declínio e à morte definitiva, enquanto parece estar ostensivamente lutando contra isso o tempo todo com suas contínuas manobras heroicas? 
Deixe-me colocar a questão de forma um tanto diferente. O que o médico moderno deveria estar fazendo? Ele deveria seja prevenir uma doença para que não aconteça, seja curá-la caso ela ocorra. O que o médico moderno, inclusive eu, está fazendo? Ele nem previne e nem cura, a não ser em poucas situações. Ele apenas controla o alastramento da doença e oferece cuidados paliativos ao fazê-lo; seu lema pode ser resumido em uma sentença: curar às vezes, confortar sempre, machucar o menos possível, causar danos nunca (Singh e Singh, 2005).

$\mathrm{O}$ epítome do tratamento paliativo, certamente, mas não aquele da cura e nem o da prevenção.

Essa percepção não impele o médico moderno em direção à cura ou à prevenção. Ela o impulsiona em direção a tratamentos paliativos mais numerosos e mais eficientes. De modo a livrá-lo rapidamente da constatação que o encara e expõe sua nudez frente a tudo, especialmente perante sua consciência.

\section{O que faremos? Eros guia, Tanatos prevalece ao final}

Então o que faremos? Seria conveniente, mas fácil, aceitar a formulação de Freud segundo a qual Tanatos e Eros são as duas pulsões que regem nossas vidas e Tanatos prevalece - e deve fazê-lo - ao final, já que a realidade definitiva é a morte, sejam quais forem as manobras que fazemos para preveni-la. O que explica por que a propulsão da medicina moderna é em direção ao controle, e não à cura ou à prevenção: trata-se apenas de uma manifestação de seu Tanatos.

Evidentemente, isso não significa que nós não exerçamos Eros, já que ele se exerce por si mesmo de qualquer forma. E os objetivos paliativos da medicina são apenas uma das manifestações desse exercício.

Esse é um Eros efetivo, porém fraco. Ao não fazer da cura e da prevenção seus objetivos, é como se a medicina aceitasse a inevitabilidade de Tanatos para invalidar seus esforços.

A medicina deve tornar-se a mais gloriosa manifestação de um Eros que previne/cura doenças e promove bem-estar em sua melhor forma possível. Isso seria um Eros efetivo e forte.

Até que a morte chegue. O que acontecerá.

Quando, por fim, Tanatos prevalecerá.

O que pode levar-nos à seguinte pergunta: se a morte deve prevalecer ao final, que sentido tem, afinal, o exercício do Eros? Por que não aceitar a realidade, parar de lutar contra Tanatos e, antes, aceitá-lo de bom grado?

A resposta é que Tanatos prevalece apenas ao final. É o ínterim que realmente importa - o intervalo entre a vida e a morte. O que cada um faz com isso está 


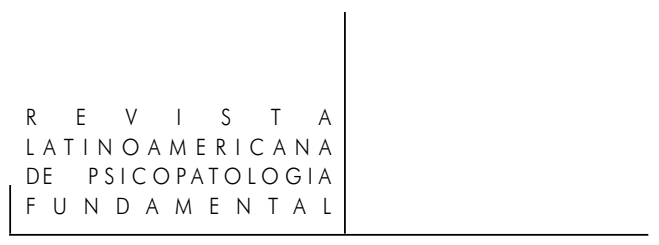

em suas mãos, e é para isso que se dirige legitimamente todo o esforço humano. Portanto, Eros deve ser consciente e vigorosamente promovido. Por que permitir que algo que deve acontecer somente ao final passe por cima do processo?

O conceito-chave é ao final. Não prematuramente, não em surdina, como ocorre atualmente devido à nossa falta de direção.

Com a compreensão de que Tanatos deve prevalecer ao final, é preciso saber quando recuar e parar de lutar, para assegurar uma saída digna. Todo o dispêndio de recursos humanos para evitar o inevitável então cessará. O papel de impotência da medicina moderna, misturado à grandiosidade que promove a vida a qualquer custo - por exemplo, em estados vegetativos persistentes - terá então fim.

Um Eros deve guiar nossas consciências e nossos esforços; mesmo ao aceitarmos a verdade de Tanatos que deve, por fim, prevalecer.

\section{Prevenção e cura como lema}

Se o aceitarmos, o caminho à nossa frente é muito claro.

\section{Medicina moderna}

Bastante progresso em doenças infecciosas e emergências $\square$ Prevenção e cura

Em outras palavras, sobretudo paliativa e voltada ao controle Farmacologia, psicofarmacologia incluída, é similar

Qual é o desafio?

(

Passagem de controle/paliação - prevenção/cura/bem-estar com longevidade Como?

प

Vacinas contra hipertensão, diabetes, câncer etc.

Papel da meditação, yoga, espiritualidade etc. na prevenção de doenças em vários níveis Estudos sobre longevidade, mudanças de estilo de vida e centenários saudáveis

Olhar para a medicina complementar e alternativa

Farmacologia, psicofarmacologia incluída, para assistir a cura/prevenção

Mudança de paradigma de controle/paliação para prevenção/cura

$\square$

Bem-estar com longevidade

(

Medicina tornar-se-á uma manifestação do Eros humano, não de seu Tanatos Q

Eros prevalece sempre, Tanatos prevalece apenas ao final

Figura 1: tabela de fluxo do artigo

Rev. Latinoam. Psicopat. Fund., São Paulo, v. 13, n. 2, p. 265-282, junho 2010 
O primeiro objetivo da medicina é velar para que ninguém tenha que chegar a um hospital ou a uma clínica. É a isso que me refiro por prevenção (tecnicamente chamada de prevenção primária; sem, é claro, excluir a importância da prevenção secundária - detecção precoce e pronto atendimento - e da prevenção terciária - restauração de funções e redução de incapacidades). Este deveria ser seu objetivo prioritário, não o último, o que significa promoção da saúde e de atividades educativas, água limpa, alimentação nutritiva, moradias limpas e em locais seguros, saneamento apropriado, controle da poluição, mitigação da pobreza, maior poder aos desprovidos de recursos, modificações de estilo de vida. Uma longa lista que envolve múltiplos agentes, não sob controle da medicina e de seus autores. Essa é uma razão fundamental que talvez explique por que isso não está no topo da agenda. E, é evidente, porque corta pela raiz a justificativa para o establishment médico e sua proliferação.

Mas a medicina está tão orientada para o tratamento quanto para a perspectiva social. Pois a saúde é um instrumento para o bem-estar, e apenas pode ser atingida para todos quando todos estiverem mobilizados para ela e tornarem-se conscientes daquilo que deveria ser seu legítimo direcionamento (Singh e Singh, 2004). Além disso, o ato de prevenir, entendido como evitar que uma doença ocorra, também significa encontrar vacinas e outras medidas preventivas contra todas as doenças, não apenas as infecciosas. Isso não deve mais soar risível. É reconfortante observar que há vacinas sendo pesquisadas, especialmente para diabetes (Phillips et al., 2008; Richardson et al., 2009), hipertensão (Mitka, 2007; Ambühl et al. 2007; Phisitkul, 2009), cânceres (especialmente cervical: Jenkins, 2008; Keam e Harper, 2008; Schwarz, 2008), e pelo menos sugeridas para esquizofrenia e para outros distúrbios mentais (Tomlinson et al., 2009).

A medicina deve ainda pesquisar e dar relevo a mudanças de estilo de vida que previnem doenças, e combater doenças relacionadas à pobreza e ao estilo de vida (Singh e Singh, 2008). Alguns trabalhos neste campo já estão sendo feitos, por exemplo, em cânceres (Anand et al., 2008), diabetes tipo 2 (Misra, 2009), doença cardiovascular (Pischke et al., 2008), colite ulcerativa (Langhorst et al., 2007) e benefícios de uma dieta vegetariana em doenças relacionadas ao estilo de vida (Segasothy e Phillips, 1999).

A psiquiatria preventiva também precisa de um impulso, para a própria psiquiatria e para a medicina em geral, uma vez que nós sabemos que o sofrimento psicológico está na raiz de problemas de saúde comuns, que alcançam clínicos de cuidados primários e complicam muitas manifestações de outros distúrbios em todos os níveis de sua manifestação. As relações complexas entre as interações genes-ambiente, particularmente a influência recíproca de fatores de vulnerabilidade e resiliência próprios à biografia de uma pessoa, requerem um exame mi- 
nucioso da psiquiatria individualizada preventiva (Müller-Spahn, 2008), assim como a redução do estigma para a prevenção secundária (Reeder e Pryor, 2008). O papel da psicologia da saúde e da medicina comportamental, seu campo correlato, que focam no entrelaçamento entre predisposições biológicas, comportamento e contexto social, também requer apoio entusiasta como um meio para promover comportamentos salutares e evitar aqueles prejudiciais à saúde (Kaplan, 2009).

A medicina moderna deve ainda olhar de perto para as asserções da medicina complementar e alternativa, incluindo yoga, meditação e espiritualidade, e não desprezá-las apenas devido às suas reivindicações grandiosas e à presença de alguns charlatões no grupo. Antes, deve submeter tais alegações a um rigoroso exame experimental e científico. Alguns estudos recentes sobre yoga em geral (Lipton, 2008; Bijlani, 2008; Corliss, 2001; Oken et al., 2007; Brown e Gerbag, 2005; Shapiro et al., 2007; Flegal et al., 2007) e yoga em cânceres (Culos-Reed et al., 2006; Bower et al., 2005; Smith e Pukall, 2009; Danhauer et al., 2009) são promissores nesse sentido. Pesquisas sobre meditação como um complemento à medicina moderna merecem atenção especial. Algumas frentes promissoras sobre longevidade e saúde por meio da meditação yogue estão em curso (Bushell, 2009), bem como sobre meditação em geral (Bushell e Thiese, 2009); práticas meditativas para a saúde (Ospina et al., 2007), e seus experimentos clínicos (Ospina et al., 2008); sudarshan kriya (técnica de respiração) em situações de estresse, ansiedade e depressão (Brown e Gerbarg, 2009); meditação transcendental e longevidade (Alexander et al., 1989); meditação e retardamento do envelhecimento (Epel et al., 2009); atenção plena (mindfulness) e sofrimento (Jain et al., 2007); e atenção plena e bem-estar (Shapiro et al., 2008). A espiritualidade e estudos científicos sobre o tema requerem igualmente exame minucioso. Algumas áreas da espiritualidade que têm interessado os pesquisadores recentemente são: emoções positivas e espiritualidade (Vaillant, 2008), sua neurobiologia (Mohandas, 2008), presença curadora (McDonough-Means et al., 2004), encontro espiritual e tratamento complementar (Foster, 2006), espiritualidade e psiquiatria (Mohr, 2006), saúde e espiritualidade no cuidado intensivo (Puchalski, 2004), espiritualidade e cuidado holístico no tratamento intensivo (Carpenter et al., 2008), dificuldade de abordar a espiritualidade no ambiente médico (Molzahn e Sheilds, 2008) etc. Para promover rigorosas pesquisas científicas sobre reivindicações da medicina complementar e alternativa (MCA), esforços notáveis estão sendo feitos por publicações relativamente novas no campo, tais como Evidence Based Complementary and Alternative Medicine (publicação da Universidade de Oxford desde junho de 2004 - http://ecam.oxfordjournals.org); BMC Complementary and Alternative Medicine (publicado pela BioMed Central desde 2001 - http://biomedcentral.com/ 
bmccomplementalternmed.org); Alternative Therapies in Health and Medicine (desde 1995, a primeira publicação do campo da medicina alternativa a ser indexada pela National Library of Medicine) - http://alternative-therapies.com); Journal of Alternative and Complementary Medicine (desde 1995, publicação oficial da International Society for Complementary Medicine Research - http:// liebertpub.com/products/product.aspx?pid=26). Alguns notáveis trabalhos relativamente recentes sobre medicina alternativa no campo da ansiedade e depressão (van der Watt et al., 2008), depressão em mulheres (Manber et al., 2002), mulheres na menopausa (Kronenberg e Fugh-Berman, 2002), distúrbios do sono na terceira idade (Gooneratne, 2008), osteoartrite (Ernst, 2006), asma (Pretorius, 2009) etc. não devem passar despercebidos.

E enquanto tudo isso acontece, os caras da medicina preventiva e social que praticam a medicina mainstream precisam acordar e melhorar seu desempenho, de forma a deixar de estar fora do jogo e salientar sua importância. E, é claro, para reenfatizar que é melhor prevenir do que remediar (Phakathi, 2009, em relação a abuso sexual infantil, mas aplicável em qualquer lugar).

\section{Cura}

O segundo objetivo da medicina é curar pacientes caso cheguem ao hospital ou a uma clínica, de modo que não tenham a mesma doença novamente. Nem outra relacionada a ela. E de modo que passem menos tempo em hospitais e mais tempo em suas casas e em seus locais de trabalho. Para tanto, a ênfase deve passar do controle para a cura, mesmo que nós saibamos que este é um objetivo distante atualmente. Distante não significa inalcançável. Na verdade, o termo distante deve deixar claro o quanto ainda precisa ser feito, e em qual direção. Eu adoraria adicionar uma lista de referências neste ponto, mas lamento encontrar muito poucas de alguma consequência.

Longevidade e bem-estar

O terceiro objetivo é estudar de perto e apresentar pesquisas em longevidade e bem-estar. Bem-estar implica: 1) presença de emoções positivas e ausência de emoções negativas; 2) traços maduros de caráter, incluindo autodirecionamento, cooperação e autotranscendência; 3) satisfação com a vida ou qualidade de vida; 4) força de caráter e virtudes como esperança, compaixão e coragem, todas as quais podem ser medidas em escalas (Cloninger, 2008). Alguma literatura relativamente recente foca em telômeros e longevidade (Haussmann e Mauck, 
2008); diferença de sexos na longevidade (Franceschi et al., 2000); imunologia e longevidade (Candore et al., 2006); fatores psicossociais e longevidade (Darviri et al., 2009) etc. O modelo do Reorganizational Healing (ROH) é um interessante trabalho recente no campo de bem-estar, mudança de comportamento, prática holística e cura (Epstein et al., 2009). Qualquer pessoa que nunca tenha estado em um hospital e tiver mais de sessenta anos é um bem precioso para pesquisas. Qualquer pessoa de mais de sessenta anos que não tenha nenhuma doença relacionada a estilo de vida é igualmente preciosa. Todos aqueles na casa dos noventa que estão fisicamente e mentalmente ativos, ainda que doentes, formam outro grupo muito precioso. E todos com mais de cem anos formam o mais precioso grupo para pesquisas. É fascinante ver a amplitude de estudos sobre esse tema. Houve uma germinação de pesquisas sobre centenários na última década - alguns dos mais interessantes e promissores estão nas áreas de centenários e envelhecimento saudável (Engberg et al. 2009); antioxidantes e centenários saudáveis (Mecocci et al., 2000); nonagenários e centenários na China (Ye et al., 2009); centenários em Bama (Xiao et al., 1996); qualidade de vida e longevidade (Jeune, 2002); centenários dinamarqueses, não necessariamente saudáveis, mas ainda autônomos (Andersen-Ranberg et al., 2001); e não necessariamente com demência (Andersen-Ranberg et al., 2006); centenários e suas funções cognitivas (Silver et al., 2001); centenários livres de demência (Perls, 2004a, 2004b); centenários agindo diferente (Perls, 2006); estados cognitivos de centenários (Luczywek et al., 2007); envelhecimento bem-sucedido de centenários: mitos e realidade (Motta et al., 2005); atividade física e centenários (Antonini et al., 2008); centenários envelhecendo com suavidade (Willcox et al., 2007) etc.

Farmacologia como ferramenta para prevenção: cura e bem-estar

A farmacologia é uma ferramenta muito potente, mas, atualmente, em sua maior parte ela é voltada para controle e tratamentos paliativos. Ela deve ser conduzida em direção à cura e à prevenção. Este é seu grande desafio, se for capaz de transcender compulsões econômicas e acordar para seu verdadeiro papel na medicina. A psicofarmacologia é similar, mas, adicionalmente, requer um auxílio maior de diagnósticos clínicos e investigativos para ajudar a psiquiatria a se tornar uma disciplina madura, deixando seu status provisório no presente (Singh e Singh, 2009). Embora tenha principalmente desenvolvido novas drogas que são mais toleráveis do que mais eficientes, ela também ajudou a reduzir o estigma e tornou médicos de cuidados primários mais confortáveis ao tratar de distúrbios mentais (Schwartz, 2010). 


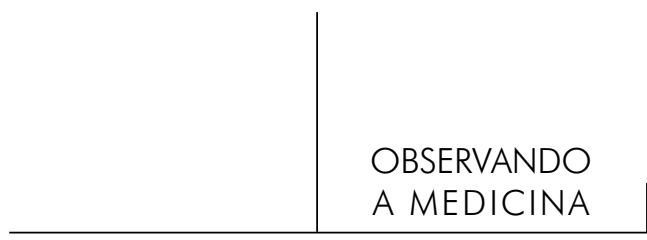

Mudança de paradigma de controle/paliação para prevenção/cura

O foco deve passar do controle/paliação para cura e prevenção. O objetivo final é longevidade com bem-estar, para o qual estar livre de doenças é um item muito importante. Financiamentos significativos para pesquisas devem guiar esses esforços, ainda que não se negligenciem maiores refinamentos na moderna medicina paliativa.

O mestre estremece ao sorrir

O trajeto está claro. O objetivo está dado.

Vamos tentar fazer o trem partir?

O outro caminho é, evidentemente, despertar com um sonho, lembrar o grande mestre e seus esforços para entender os fundamentos da doença ao falar da análise de sonhos e do inconsciente, e calar a compreensão com a negação que é tão característica de grande parte da moderna medicina, bem como da farmacologia e psicofarmacologia. E cair em um sono que empurra sonhos desconfortáveis para reinos que trabalham para energizar Tanatos e impelir o homem ao seu inevitável fim.

Você decide o que fazer.

$\mathrm{O}$ mestre abre um sorriso zombeteiro enquanto se revira com dor em sua sepultura.

\section{Notas conclusivas}

1. Passemos do caminho da paliação e controle para o da prevenção e da cura. Primeiramente, vamos pensar que isso é possível.

2. Esse é o futuro objetivo da farmacologia, incluindo a psicofarmacologia, e também da pesquisa e prática médicas.

3. Para assegurar longevidade com bem-estar, não vamos descartar o trabalho da medicina complementar e alternativa, mas antes submetê-lo a rigoroso exame científico.

4. Precisamos ainda promover estudos sobre a longevidade.

Leve a mensagem para casa

Uma mudança de paradigma na medicina tradicional passando do controle e paliação para a prevenção e cura deve prevalecer. O objetivo final é longevidade com bem-estar. 


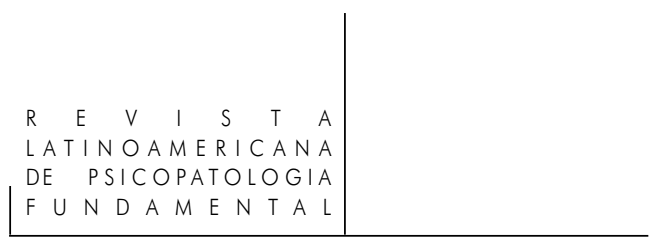

\section{Referências}

Alexander, C.N.; Langer, E.J.; Newman, R.L.; Chandler, H.M.; Davies, J.L. Transcendental meditation, mindfulness, and longevity: an experimental study with the elderly. $J$ Pers Soc Psychol, v. 57, n. 6, p. 950-964, 1989.

AmbüHl, P.M.; Tissot, A.C.; Fulurija, A.; Maurer, P.; Nussberger, J.; Sabat, R.; Nief, V.; Schellekens, C.; Sladko, K.; Roubicek, K.; Pfister, T.; Rettenbacher, M.;Volk, H.D.;

Wagner, F.; Müller, P.; Jennings, G. T.; Bachmann, M.F. A vaccine for hypertension based on virus-like particles: preclinical efficacy and phase I safety and immunogenicity. J Hypertens, v. 25, n. 1, p. 63-72, 2007.

Anand, P.; Kunnumakkara, A.B.; Sundaram, C.; Harikumar, K.B.; Tharakan, S.T.; Lai, O.S.; Sung, B.; AgGarwal, B.B. Cancer is a preventable disease that requires major lifestyle changes. Pharm Res, v. 25, n. 9, p. 2097-2116, 2008.

Andersen-Ranberg, K.; Schroll, M.; Jeune, B. Healthy centenarians do not exist, but autonomous centenarians do: a population-based study of morbidity among Danish centenarians. J Am Geriatr Soc, v. 49, n. 7, p. 900-908, 2001 a.

Andersen-Ranberg, K.; VasegaArd, L.; Jeune, B. Dementia is not inevitable: a population-based study of Danish centenarians. J Gerontol B Psychol Sci Soc Sci, v. 56, n. 3, p. 152-159, 2001b.

Antonini, F.M.; Magnolfi, S.U.; Petruzzi, E.; Pinzani, P.; Malentacchi, F.; Petruzzi, I.; MAsotTI, G.; IMUSCE. Physical performance and creative activities of centenarians. Arch Gerontol Geriatr, v. 46, n. 2, p. 253-261. Epub 2007 Jun , 2008.

BiJlani, R.L. Yoga: an ancient tool in modern medicine. Natl Med J India, v. 21, n. 5, p. 215-216, 2008.

Bower, J.E.; Woolery, A.; Stemlieb, B.; Garet, D. Yoga for cancer patients and survivors. Cancer Control, v. 12, n. 3, p. 165-171, 2005.

Brown, R.P.; Gerbarg, P.L. Sudarshan Kriya Yogic breathing in the treatment of stress, anxiety, and depression. Part II - Clinical applications and guidelines. J Altern Complement Med, v. 11, n. 4, p. 711-717, 2005.

Brown, R.P.; Gerbarg, P.L. Yoga breathing, meditation, and longevity. Ann N Y Acad Sci, n.1172, p. 54-62, 2009.

Bushell, W.C. Longevity: potential life span and health span enhancement through practice of the basic yoga meditation regimen. Ann N Y Acad Sci, n. 1172, p. 20-27, 2009. Bushell, W.C.; THEISE, N.D. Toward a unified field of study: longevity, regeneration, and protection of health through meditation and related practices, Ann N Y Acad Sci, n. 1172, p. 5-19, 2009.

Candore, G..;Balistreri, C.R.; Listi, F.; Grimaldi, M.P.; Vasto, S.; Colonna-Romano, G..; Franceschi, C.; Lio, D.; Caselli, G.; Caruso, C. Immunogenetics, gender, and longevity. Ann N Y Acad Sci, n. 1089, p. 516-537, 2006.

CARPenter, K.; Girvin, L.; Kitner, W.; Ruth-SAHD, L.A. Spirituality: a dimension of holistic critical care nursing. Dimens Crit Care Nurs, v. 27, n. 1, p. 16-20, 2008. 
Cloninger, C.R. On Well-Being: Current Research Trends And Future Directions (Ed), $M S M$, v. 6, n. 1, p. 3-9, 2008.

Corliss, R. The power of yoga. Time, Apr 23, ano 157, n. 16, p. 54-63, Apr. 23, 2001. Culos-Reed, S.N.; Carlson, L.E.; Daroux, L.M.; Hately-Aldous, S. A pilot study of yoga for breast cancer survivors: physical and psychological benefits. Psychooncology, v. 15, n. 10, p. 891-897, 2006.

Danhauer, S.C.; Mihalko, S.L.; Russell, G.B.; Campbell, C.R.; Felder, L.; Daley, K.; LEvINE, E.A. Restorative yoga for women with breast cancer: findings from a randomized pilot study. Psychooncology, v. 18, n. 4, p. 360-368, 2009.

Darviri, C.; Demakakos, P.; Tigani, X.; Charizani, F.; Tsiou, C.; Tsagkari, C.;

ChLIAOUTAKIs, J.; Monos, D. Psychosocial dimensions of exceptional longevity: a qualitative exploration of centenarians' experiences, personality, and life strategies. Int $J$ Aging Hum Dev, v. 69, n. 2, p. 101-118, 2009.

Engberg, H.; Oksuzyan, A.; Jeune, B.; Vaupel, J.W.; Christensen, K. Centenarians - a useful model for healthy aging? A 29-year follow-up of hospitalizations among 40,000 Danes born in 1905. ing Cell, v. 8, n. 3, p. 270-276. Epub 2009 Mar 27, 2009.

Epel, E.; Daubenmier, J.; Moskowitz, J.T.; Folkman, S.; Blackburn, E. Can meditation slow rate of cellular aging? Cognitive stress, mindfulness, and telomeres. Ann N Y Acad Sci, n. 1172, p. 34-53, 2009.

Epstein, D.M.; Senzon, S.A.; Daniel Lemberger, D.A. Reorganizational Healing: A Paradigm for the Advancement of Wellness, Behavior Change, Holistic Practice, and Healing. Jr Altern Complement Med, v. 15, n. 5, p. 475-487, 2009.

ERNST, E. Complementary or alternative therapies for osteoarthritis. Nat Clin Pract Rheumatol., v. 2, n. 2, p. 74-80, 2006.

Flegal, K.E.; Kishiyama, S.; Zajdel, D.; HaAs, M.; Oken, B.S. Adherence to yoga and exercise interventions in a 6-month clinical trial. BMC Complement Altern Med, n. 7, p. 37, 2007.

FoSTER, E. The spiritual encounter within a complementary therapy treatment. Complement Ther Clin Pract, v. 12, n. 2, p. 163-169. Epub 2005 Oct 5, 2006.

Franceschi, C.; Motta, L.; Valensin, S.; Rapisarda, R.; Franzone, A.; Berardelli, M.; Motta, M.; Monti, D.; Bonafè, M.; Ferrucci, L.; Deiana, L.; Pes, G.M.; Carru, C.; Desole, M.S.; Barbi, C.; Sartoni, G.; Gemelli, C.; Lescai, F.; Olivieri, F.; Marchegiani, F.; Cardelli, M.; Cavallone, L.; Gueresi, P.; Cossarizza, A., Troiano, L.; Pini, G.; Sanson,, P.; Passeri, G.; Lisa, R.; Spazzafumo, L.; Amadio, L.; Giunta, S.; Stecconi, R.; Morresi, R.; Viticchi, C.; Mattace, R.; De Benedictis, G.; Baggio, G. Do men and women follow different trajectories to reach extreme longevity? Italian Multicenter Study on Centenarians (IMUSCE). Aging (Milano), v. 12, n. 2, p. 77-84, 2000.

GoONERATNE, N.S. Complementary and alternative medicine for sleep disturbances in older adults. Clin Geriatr Med, v. 24, n. 1, p. 121-38, viii, 2008.

HaUSSMANn ,M.F.; MaUCK, R.A. Telomeres and longevity: testing an evolutionary hypothesis. Mol Biol Evol, v. 25, n. 1, p. 220-228. Epub 2007 Dec 10, 2008. 


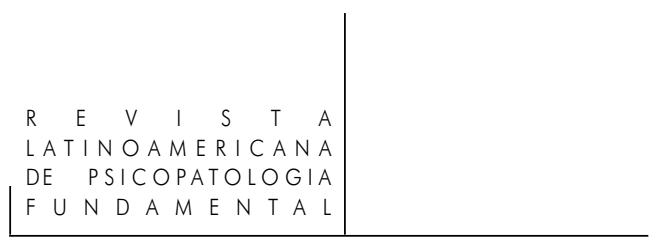

Jain, S.; ShaPiro, S.L.; Swanick, S.; Roesch, S.C.; Mills, P.J.; Bell, I.; Schwartz, G.E. A randomized controlled trial of mindfulness meditation versus relaxation training: effects on distress, positive states of mind, rumination, and distraction. Ann Behav Med, v. 33, n. 1, p. 11-21, 2007.

JENKINS, D. A review of cross-protection against oncogenic HPV by an HPV-16/18 AS04adjuvanted cervical cancer vaccine: importance of virological and clinical endpoints and implications for mass vaccination in cervical cancer prevention. Gynecol Oncol, n. 110 (3 Suppl 1), p. 18-25, 2008.

JEUne, B. Living longer - but better? Aging Clin Exp Res, v. 14, n. 2, p. 72-93, 2002.

KaPLAN, R.M. Health Psychology: Where Are We And Where Do We Go From Here? MSM, n. 7, p. 3-9, 2009.

KeAM, S.J.; HARPER, D.M. Human papillomavirus types 16 and 18 vaccine (recombinant, AS04 adjuvanted, adsorbed) [Cervarix]. Drugs, v. 68, n. 3, p. 359-372, 2008.

Kronenberg, F.; Fugh-Berman, A. Complementary and alternative medicine for menopausal symptoms: a review of randomized, controlled trials. Ann Intern Med, v. 137, n. 10, p. 805-813, 2002.

Langhorst, J.; Mueller, T.; Luedtke, R.; Franken, U.; Paul, A.; Michalsen, A.;

Schedlowski, M.; Dobos, G.J.; Elsenbruch, S. Effects of a comprehensive lifestyle modification program on quality-of-life in patients with ulcerative colitis: a twelve-month follow-up. Scand J Gastroenterol, v. 42, n. 6, p. 734-745, 2007.

278 LiPTON, L. Using yoga to treat disease: an evidence-based review. JAAPA, v. 21, n. 2, p. 34-36, 38, 41, 2008.

Luczywek, E.; Gabryelewicz, T.; Barczak, A.; Religa, D.; Pfeffer, A.; Styczynska, M.; Peplonska, B.; Chodakowska-Zebrowska, M.; Barcikowska, M. Neurocognition of centenarians: neuropsychological study of élite centenarians. Int J Geriatr Psychiatry, v. 22, n. 10, p. 1004-1008, 2007.

McDonough-Means, SI.; Kreitzer, M.J.; Bell, I.R. Fostering a healing presence and investigating its mediators. J Altern Complement Med, n. 10, Suppl 1, p. S25-41, 2004.

MAnber, R.; Allen, J.J.; Morris, M.E. Alternative treatments for depression: empirical support and relevance to women. J Clin Psychiatry, v. 63, n. 7, p. 628-640, 2002.

Mecocci, P.; Polidori, M.C.; Troiano, L.; Cherubini, A.; Cecchetti, R.; Pini, G.;

Straatman, M.; Monti, D.; Stahl, W.; Sies, H.; Franceschi, C.; Senin, U. Plasma antioxidants and longevity: a study on healthy centenarians. Free Radic Biol Med, v. 28. n. 8, p. 1243-1248, 2000.

MisRa, A. Prevention of type 2 diabetes: the long and winding road. The Lancet, n. 374 (9702), p. 1655-1656, 2009.

MiтKA, M. Vaccine takes aim at hypertension. JAMA, v. 298, n. 24, p. 2854-2855, 2007.

MoHANDAS, E. Neurobiology of Spirituality. MSM, v. 6, n. 1, p. 63-80, 2008.

MонR, W.K. Spiritual issues in psychiatric care. Perspect Psychiatr Care, v. 42, n. 3, p. 174-183, 2006.

Molzahn, A.E.; SheILDs, L. Why is it so hard to talk about spirituality? Can Nurse, v. 104, n. 1, p. 25-29, 2008. 
Motta, M.; Bennati, E.; Ferlito, L.; Malaguarnera, M.; Motta, L.; Italian Multicenter Study on Centenarians (IMUSCE). Successful aging in centenarians: myths and reality. Arch Gerontol Geriatr, v. 40, n. 3, p. 241-51, 2005.

MüLlER-Spahn, F. Individualized preventive psychiatry: syndrome and vulnerability diagnostics, Eur Arch Psychiatry Clin Neurosci, n. 258, Suppl 5, p. 92-97, 2008.

OKen, B.S.; Zajdel, D.; Kishiyama, S.; Flegal, K.; Dehen, C.; HaAs, M.; Kraemer, D.F.; LAWRENCE, J.; LEYVA, J. Randomized, controlled, six-month trial of yoga in healthy seniors: effects on cognition and quality of life. Altern Ther Health Med, v. 12. n. 1, p. 40-47, 2006.

Ospina, M.B.; Bond, K.; Karkhaneh, M.; Tuosvold, L.; Vandermeer, B.; Liang, Y.; Bialy, L.; Hooton, N.; Buscemi, N.; Dryden, D.M.; Klassen, T.P. Meditation practices for health: state of the research. Evid Rep Technol Assess (Full Rep), n. 155, p. 1-263, 2007. Ospina, M.B.; Bond, K.; Karkhaneh, M.; Buscemi, N.; Dryden, D.M.; Barnes, V.; CARLSOn, LE.; DuseK, J.A.; ShannAhoff-Khalsa, D. Clinical trials of meditation practices in health care: characteristics and quality. J Altern Complement Med, v. 14, n. 10, p. 11991213, 2008.

Perls, T. Dementia-free centenarians. Exp Gerontol, v. 39, n. 11-12, p. 1587-1593, 2004a. . Centenarians who avoid dementia. Trends Neurosci, v. 27, n. 10, p. 633-636, $2004 b$.

PerLs, T.T. The different paths to 100. Am J Clin Nutr, v. 83, n. 2, p. 484S-487S, 2006. Pнакатн, M. Prevention is better than cure. Bull World Health Organ, v. 87, n. 5, p. 328329, 2009.

Phillips, B.; Nylander, K.; Harnaha, J.; Machen, J.; Lakomy, R.; Styche, A.; Gillis, K.; Brown, L.; Lafreniere, D.; Gallo, M.; Knox, J.; Hogeland, K.; Trucco, M.;

GiannoukaKis, N. A microsphere-based vaccine prevents and reverses new-onset autoimmune diabetes. Diabetes, v. 57, n. 6, p. 1544-1555, 2008.

Phisitkul, S. CYT-006-AngQb, a vaccine against angiotensin II for the potential treatment of hypertension. Curr Opin Investig Drugs, v. 10, n. 3, p. 269-275, 2009.

PischKe, C.R.; Scherwitz, L.; WeIDNER, G.; Ornish, D. Long-term effects of lifestyle changes on well-being and cardiac variables among coronary heart disease patients. Health Psychol, v. 27, n. 5, p. 584-592, 2008.

PRETORIUS, E. The role of alternative and complementary treatments of asthma. Acupunct Electrother Res, v. 34, n. 1-2, p. 15-26, 2009.

PuCHALSKI, C. Spirituality in health: the role of spirituality in critical care. Crit Care Clin, v. 20, n. 3, p. 487-504, x, 2004.

Reeder, G.D.; Pryor, J.B. Dual Psychological Processes Underlying Public Stigma and the Implications for Reducing Stigma. MSM, v. 6, n. 1, p. 175-186, 2008.

Richardson, S.J.; Willcox, A.; Bone, A.J.; Foulis, A.K.; Morgan, N.G. The prevalence of enteroviral capsid protein vp1 immunostaining in pancreatic islets in human type 1 diabetes. Diabetologia, v. 52, n. 6, p. 1143-1151, 2009.

SCHWARZ, T.F. AS04-adjuvanted human papillomavirus-16/18 vaccination: recent ad- 


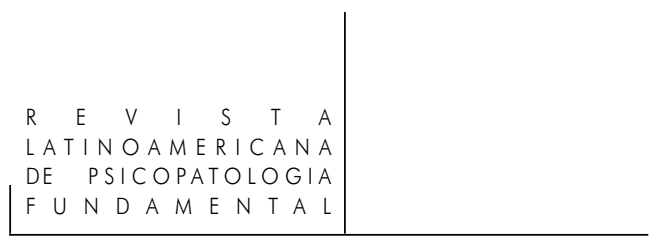

vances in cervical cancer prevention. Expert Rev Vaccines, v. 7, n. 10, p. 1465-1473, 2008. SchwarTz, T.L. Psychopharmacology today: Where are we and where do we go from here? (Ed). MSM, n. 8, 2010 [In press].

Segasothy, M.; Phillips, P.A. Vegetarian diet: panacea for modern lifestyle diseases? $Q J$ Med, n. 92, p. 531-544, 1999.

Shapiro, D.; CoOK, I.A.; Davydov, D.M.; Ottaviani, C.; Leuchter, A.F.; Abrams, M. Yoga as a Complementary Treatment of Depression: Effects of Traits and Moods on Treatment Outcome. Evid Based Complement Alternat Med., v. 4, n. 4, p. 493-502, 2007.

Shapiro, S.L.; Oman, D.; Thoresen, C.E.; Plante, T.G.; Flinders, T. Cultivating mindfulness: effects on well-being. J Clin Psychol, v. 64. n. 7, p. 840-862, 2008.

Silver, M.H.; JilinsKaia, E.; Perls, T.T. Cognitive functional status of age-confirmed centenarians in a population-based study. J Gerontol B Psychol Sci Soc Sci, v. 56. n. 3, p. 134-140, 2001.

SingH, A.R.; SingH, S.A. Health for all, All for health: Clich?, Dream or Commitment. $M S M$, v. 3, n. 2, p. 15-18, 2004.

; __ . Emphasising prevention, developing therapies, complementing approaches. $M S M$, v. 3, n. 2, p. 15-18, 2005.

; _ . To cure sometimes, to comfort always, to hurt the least, to harm never. $M S M$, v. 4, p. 8-9, 2006.

; _ . Diseases of Poverty and Lifestyle, Well-Being and Human Development. $M S M$, v. 6, n. 1, p. 187-225, 2008.

p. 128-183, 2009.

. Notes on a few issues in the philosophy of psychiatry. $M S M$, v. 7, n. 1,

SMITH, K.B.; PuKall, C.F. An evidence-based review of yoga as a complementary intervention for patients with cancer. Psychooncology, v. 18, n. 5, p. 465-475, 2009.

Tomlinson, M.; Rudan, I.; SaXena, S.; Swartz, L.; Tsai, AC.; Patel, V. Setting priorities for global mental health research. Bull World Health Organ, v. 87, n. 6, p. 438-446, 2009. Vaillant, G.E. Positive Emotions, Spirituality and the Practice of Psychiatry. MSM, v. 6, n. 1, p. 48-62, 2008.

van DeR Watt, G..; Laugharne, J.; JAnCA, A. Complementary and alternative medicine in the treatment of anxiety and depression. Curr Opin Psychiatry, v. 21, n. 1, p. 37-42, 2008. Willcox, D.C.; Willcox, B.J.; Shimajiri, S.; KuReChi, S.; Suzuki, M. Aging gracefully: a retrospective analysis of functional status in Okinawan centenarians. Am J Geriatr Psychiatry, v. 15, n. 3, p. 252-256, 2007.

XIAO, Z.; XU, Q.; YUAN, Y. Solving the mystery of the status and longevity of centenarians in Bama. Chin J Popul Sci, v. 8, n. 4, p. 385-394, 1996.

Ye, J.J.; LI, J.C.; PenG, L.; Gong, Y.Y.; XIE, L.; Lian, S.G.; YANG, Z.; ZhOU, Y.F. Nonagenarians and centenarians in a rural Han Chinese population: lifestyle and epidemics. J Am Geriatr Soc, v. 57, n. 9, p. 1723-1724, 2009. 
(Modern Medicine: Towards prevention, cure, well-being and longevity)

Modern medicine has done much in the fields of infectious diseases and emergencies to aid cure. In most other fields, it is mostly control that it aims for, which is another name for palliation. Pharmacology, psychopharmacology included, is mostly directed towards such control and palliation too. The thrust, both of clinicians and research, must now turn decisively towards prevention and cure. Also, longevity with well-being is modern medicine's other big challenge. Advances in vaccines for hypertension, diabetes, cancers etc, deserve attention; as also, the role of meditation, yoga, spirituality etc in preventing disease at various levels. Studies on longevity, life style changes and healthy centenarians deserve special scrutiny to find what aids longevity with wellbeing. A close look at complementary and alternative medicine is needed to find any suitable models they may have, cutting aside their big talk and/or hostility towards mainstream medical care. Medicine is a manifestation of the human eros, and should not become a means of its thanatos. It must realise its true potential, so that eros prevails, and thanatos prevails only ultimately, not prematurely.

Key words: Medicine, Eros, Thanatos, disease, prevention, cure, palliation, longevity, wellbeing, centenarians, meditation, yoga, complementary and alternative medicine, pharmacology, psychopharmacology

(La Médecine Moderne: vers la Prévention, la Guérison, le Bien-être et la Longévité) La médecine moderne a fait de grands progrès dans les domaines des maladies infectieuses et des secours médicaux. Par contre, dans la plupart des autres domaines, y compris la pharmacologie et la psychopharmacologie, elle vise surtout les soins palliatifs. Dorénavant, les cliniciens et chercheurs devront se concentrer sur la prévention et la guérison, ainsi que sur le vieillissement en bonne santé. Les progrès dans les domaines des vaccins contre l'hypertension, le diabète, le cancer, etc. méritent une attention particulière ainsi que le rôle de la méditation, due yoga et de la spiritualité dans la prévention des maladies à différents niveaux. Les études sur la longévité, les changements de style de vie et les centenaires en bonne santé sont essentielles pour découvrir les facteurs qui influencent le vieillissement en bonne santé. Les médecines complémentaires et alternatives doivent être examinées en détail pour trouver des modèles appropriés. La médecine est une manifestation de l'éros humain et ne doit pas passer au service du thanatos. Elle doit réaliser son véritable potentiel, de sorte que éros puisse prévaloir et que thanatos ne l'emporte qu'en dernière instance, et non pas de façon prématurée.

Mots clés: Médecine, Eros, Thanatos, maladie, prévention, traitement, soins palliatifs, longévité, le bien-être, centenaires, méditation, yoga, médecines complémentaires et alternatives, pharmacologie, psychopharmacologie 
(Medicina moderna: en direción a la prevención, a la cura, al bienestar y a la longevidad)

La medicina moderna ha dado mucho para ayudar a curar en el campo de las enfermedades infecciosas y de emergencias. En la mayor parte de los otros campos, ella visa, sobretodo, el control, que é otro nombre para el tratamiento paliativo. La farmacología, dentro de la cual se incluye la psicofarmacología, es igualmente dirigida al controle y al alivio. Es tiempo de profesionales del área e investigadores volcarse decisivamente para la prevención y la cura. Además, el otro grande desafío de la medicina moderna es la longevidad aliada al bienestar. Adelantos en vacunas contra la hipertensión, diabetes, cáncer etc. merecen atención, tanto como el papel de la meditación, yoga, espiritualidad etc., en la prevención de enfermedades en varios niveles. Investigaciones sobre longevidad, mudanzas en el estilo de vida y la existencia de centenarios saludables merecen un examen especialmente minucioso en la búsqueda de los factores que contribuyen para la longevidad con bienestar. Una mirada más próxima para la medicina complementaria y alternativa es necesaria para encontrar modelos apropiados que esta pueda llegar a tener, dejando de lado los grandes discursos y/o su hostilidad para con el cuidado médico tradicional. La medicina es una manifestación del Eros humano, y no debe tornar-se instrumento de su Tánatos. Ella debe realizar su verdadero potencial, de forma a que Eros prevalezca y Tánatos prevalezca apenas finalmente, no prematuramente.

Palabras-claves: Medicina, Eros, Tánatos, enfermedad, prevención, cura, paliativo,

omplementaria y alternativa, farmacología, psicofarmacología

\section{Mônica TeixeIra}

Jornalista; psicanalista; coordenadora geral do programa Universidade Virtual do Estado de São Paulo na TV Cultura (São Paulo, SP, Brasil); diretora de redação do boletim eletrônico Inovação Unicamp (Campinas, SP, Brasil).

Av. Higienópolis, 318/13

01238-001 São Paulo, SP, Brasil

e-mail: armonica@uol.com.br

AJai R. Singh, M.D.

Psiquiatra e editor do website Mens Sana Monographs (http://www.msmonographs.org). Ele tem escrito extensivamente sobre questões relacionadas à psiquiatria, filosofia, bioética, medicina e indústria farmacêutica.

14, Shiv Kripa

Trimurty Road

Nahur, Mulund

Mumbai 400080

e-mail: mensanmonographs@yahoo.co.uk 